\title{
Sport Science: Ontological and Methodological Considerations
}

Authors' contribution:

A) conception and design of the study

B) acquisition of data

C) analysis and interpretation of data

D) manuscript preparation

E) obtaining funding

\author{
Jernej Pisk
}

St. Stanislav's Institution in Ljubljana, Slovenia

ABSTRACT

Sport is the research object of variety of sciences. But, what is sport and how to think it? The aim of this article is to give some basic thoughts about the nature of sport and to present, confront and evaluate different concepts of science with their different rival approaches and understanding of sport. In general three major groups of sciences can be differentiated: natural sciences and social sciences which share common quantitative (empirical and mathematical based) methodology of research, and human sciences or humanities with their method of reflection (the analysis of concepts and rational argumentation) which go beyond empirically measurable things. Because of different scientific approaches, different understanding and concepts of sport arise which try to prevail over in society. Our comprehension of sport is therefore greatly influenced by pre-accepted methodological position. If sport is equated with physical human body movement, then natural science with its empirical methodology seems to be adequate way for cognition of sport. For social scientists sport has important role in society, therefore it cannot be reduced to mere "body movement". But humanists would say that sport is more than "body in movement" with influence in society: sport is a powerful idea or concept which needs a special unempirical method of research. Therefore human sciences with their rational reflection of human (personal) experiences can reveal us additional, but yet familiar dimensions of sport. Although their method is not empirically objective, they can deal with important life matters, moreover, their "a priori" qualitative approach to sport can give meaning and make sense out of sport, reflect about the aim and purpose of sport as well as make some ethical considerations about sport. In the article some examples are given and some problems regarding reduction of sport science to just one scientific approach are considered.

KEYWORDS sport, sport science, philosophy of sport, methodology of sport science, ontology of sport

\section{Introduction}

Sport is interesting phenomenon: on one hand it is so easy comprehensible that almost anybody is interested in sport and knows much about sport, while on the other hand it is so complex that variety of 
sciences and scientific approach deal with sport, however neither of them can fully comprehend and explain it. General expertise about sport is presented in saying that "everyone is expert about football!", and if we listen to the conversations of spectators we see that similar hold for any other sport as well. Why this is the case? In what follows some thoughts will be given regarding the nature of sport, and different methodological approaches toward sport are presented and evaluated. Every method has its own understanding of sport, and that is the reason for competition among different concepts of sport in modern society for their predomination in public understanding of sport.

\section{The power of methodology}

It is known that the object of research and method of research are in tight connection: it is not possible to see voices or hear colours. Colours can be seen and voices can be heard. Similar principle is valid elsewhere, also in the sphere of sport research. So our first questions should be: What is sport and what method of research is appropriate for it? In the field of sport science it seems to be still a confusion regarding the method of research. In some other sciences the methodological approach is not such a big problem because their object of research is much more narrow and much less complex phenomenon. The biologist who is engaged in research of plants use the methods which the best correspond to the object of their research. Because the object is something physical, they use physical methods. Similarly the mathematics use mathematical methods, which is the most appropriate for its field. But in the field of sport science the object is nothing less than human being in motion. Because sport somehow "contain" a human being with all variety of his characteristics, it can not be reduced to mere physical movement. Therefore the coexistence of different methodological approaches is needed. As elsewhere in modern world also in sport science the domination of natural and social science approach can be recognized. The method of natural and social sciences can be presented as a combination of empirical (measurement) and mathematical (calculation). But, is the quantitative scientific method of natural and social sciences sufficient to fit the object of our research, which is sport? Hosta is right when he affirms that the question "how to think sport?" is probably the most important question to consider before we enter into the research of sport (Hosta, 2005, p. 184). The question about the methodological approach toward sport is important because methodology or "technology of research" is nowadays not only the instrument how to approach toward researching object (e.g. sport), but it is the power which in great manner transform the reality which we are trying to research. Today technics in sport is not just a means for improvement of sport, but is a power which is defining what sport is and how people understand it. It is something completely different if we talk about sport in physical categories like seconds, meters, kilograms, calories, watts etc. than in categories of game, play, challenges, cooperation, virtues etc. Therefore for natural scientist sport can be something completely different than for social or human scientist. But, who is right? It seems that this situation can be compared with the poet and joiner going into forest: both staring at the same tree they will not see the same thing: the joiner will see the furniture... Nevertheless, one concept of sport is more widely accepted in society, while others are less accepted. Therefore the question is not what we can achieve in sport using sport science, but what (different methodological approaches of) sport science will make out of sport?

\section{Three braches of sciences}

Aristotle (384-322 BC) already distinguished three main branches of sciences by their ends or objects. Contemplative sciences are concerned with knowledge for its own sake; practical sciences with good action; and productive sciences with making useful or beautiful objects (Aristotle, Topics VI.6.145a14-16; Metaphysics VI.1.1025b24, XI.7.1064a16-19; Nicomachean ethics VI.2.1139a26-8). In their nature the third one are purely empirical and descriptive, the second one are normative or prescriptive and the first one are contemplative. If we compare Aristotle's branches of sciences with the field of sport science today, we can recognize that the main part of it belongs to the productive sciences: this are all those scientific attempts that try to help in sport training, in transforming of human body for the purpose of progress of performances and success in competition. The next branch of science - the practical science - is possible to recognize in 
sport education, ethics and politics. Its purpose is to give intellectual or ideological support to different kinds of sport activities (like sport for education, sport for health, sport for building national consciousness or state belonging etc.). For Aristotle the first and the most important branch of science is contemplative science, because it is the only independent science, not enslaved to external purpose, however it is often neglected in contemporary sport science because of its unpractical and unprofitable nature.

Nowadays science can be defined as:

"socially organized and systematic search for and transmission of coherent knowledge in any domain” (Hoyrup, 2000, p. 7).

For the reason of clarity it is useful to make some generalizations, so we decided to divide sciences into three main branches: ${ }^{1}$ natural sciences (like biomechanics and physiology), social sciences (like sociology) and human sciences (like philosophy). All are sciences in full right. All three branches have in common a desire to gain knowledge, to get as close as possible to truth. All have their object and their method. Natural sciences and social sciences distinguish in object of research, but have a common method of research. But social sciences and human sciences have (more or less) common object of research, but distinguish in their method of research. This classification of sciences has, in principle, instrumental character to know what can and what cannot be expected from either of sciences.

Table 1. Object and methods of natural, social and human sciences

\begin{tabular}{|l|l|l|}
\hline NATURAL SCIENCES & OBJECTS & METHODS \\
\hline SOCIAL SCIENCES & Nature... & $\begin{array}{l}\text { Empirical and mathematical } \\
\text { (measure and calculation) "from } \\
\text { outside". }\end{array}$ \\
\hline HUMAN SCIENCES & Human being, society... & $\begin{array}{l}\text { Empirical and mathematical »from } \\
\text { outside«. }\end{array}$ \\
\hline
\end{tabular}

\section{Two types of reason}

In the centre of natural and social sciences is method with its modern conception of reason. It is based on a:

"synthesis between Platonism (Cartesianism) and empiricism, a synthesis confirmed by the success of technology. On the one hand it presupposes the mathematical structure of matter, its intrinsic rationality, which makes it possible to understand how matter works and use it efficiently /.... On the other hand, there is nature's capacity to be exploited for our purposes, and here only the possibility of verification or falsification through experimentation can yield decisive certainty" (Ratzinger, 2006).

Only the kind of certainty resulting from the interplay of mathematical and empirical elements can be considered scientific. Anything that would claim to be science must be measured in accordance with this criterion, hence also sport science attempts to conform itself to this canon of scientificity.

On the other hand human sciences or humanities do not reduce themselves to empirical and mathematical explanation. The humanities investigate human actions, which differ from mere movements by having a meaning and which can only be described adequately if the purpose intended by the actor is

\footnotetext{
${ }^{1}$ Although already Aristotle distinguished three main branches of sciences, his division differ from modern one: For Aristotle the "differentia specifica" is the purpose or aim of sciences, but modern division is grounded on different object and method of research.

${ }^{2}$ Also called etic approach.

${ }^{3}$ Also called emic approach.
} 
considered. Therefore human sciences use wider understanding of reason: we as human beings can not only measure and calculate, but also understand concepts and make judgements based on reasoning. Kreeft summarizes wider reason in three acts of the mind:

1. Simple apprehension;

2. Judging;

3. Reasoning.

The mental products produced in the mind by the three acts of the mind are:

1. Concepts (the product of conceiving);

2. Judgments (the product of judging);

3. Arguments (the product of reasoning, or arguing) (Kreeft, 2008, p. 28).

As a human beings we can:

1. Comprehend nonphysical essences of things, so we can conceive the concept of sport;

2. Then, we can make a judgement about sport, e.g. sport is human bodily activity;

3. Finally, we can reason about sport and make an argument, e.g. human bodily activities are good for health, and sport is human bodily activity, therefore sport is good for health.

It is obvious that it is not possible to come to this conclusion via empirical measurement or calculation. Understanding all this is exclusive human capability of his wide reason. The book does not know what is written in it; the computer does not understand the meaning of the data that it is processing. Personal life experience is first source of meaning for us. The humanities deal with human phenomena which we understand from the inside (Hoyrup, 2000, p.167). We can only understand human from the position of being human (Ryal, 2011, p.172). We are within the flow of life which is at the same time our object of research. It is not possible to step out of life and objectively valuate/observe it, as it is possible in the case of object of natural sciences. Therefore the method of research must be different: the essence of method is observation and reflection on different phenomena in life. Its tool is analysis of concepts and rational argumentation. Also historical sources and texts are useful sources to understand the intention of human action and the meaning of it. Human sciences in sport therefore do not concentrate on efficient causes and causal explanation but on final causes and oughts. To put it short: The object of human science in sport can be anything that cannot be measured empirically or calculated mathematically; any concept or idea from sport that people can rationally deliberate about it.

Different methodological approaches results in different concepts, different understanding of sport among people. Because concepts of sport are different, all dealing with the same phenomenon - sport - , they are in a kind of competition for predomination. The strongest one will have the greater influence for the place and future understanding of sport in society. But, where any of the concepts of sport will lead us? And which of the presented methods is proper for the research of sport? To answer this question we must first know and define what is sport. Only after we analyse the concept and idea of sport, it will be possible to evaluate different research methods for researching sport.

\section{What is sport?}

First we can recognize that there is no sport as a being per se. Sport do not exist by itself (on its own), as chair, table, house, ball or human being do. Sport is always connected with somebody who "play" or "do" sport and who can exist by itself. This being who "plays" or "does" sport is human being - is person. Because sport as such is not something physical we must recognize that sport cannot be measured empirically or calculated mathematically: It is not possible to describe sport in the categories of physical variables and numbers like we can describe physical objects when we measure them, or like we put in mathematical formulae the laws of physical nature. It is only possible to measure physical human body and its movement. But sport as such is a concept, an idea about specific human bodily movement. When we think about sport, we think about this movement which has some special purpose. But purpose, as we have seen, 
cannot be measured empirically and is in domain of humanistic research. If the scientific method of natural and social science would be completely adequate for researching sport, than the presumption that sport itself is something physical, something that exists on itself and that can be measured, must be true. But sport itself is nothing physical and cannot be empirically measured or divided like we can divide physically existing object, e.g. piece of bread. Sport is a concept or an idea which is tightly connected with human person, and this shows us that just empirical and mathematical research of natural and social sciences are insufficient (McFee 2004, p. 5). Sport concerns a person: human beings play and do sports, watch sports, understand sports and judge sports. Sport only has meaning for us, but not for itself. However, we can empirically measure and calculate the facts about human body and its movement in sport: speed, power, time etc.

\section{Applying natural, social and humanistic scientific methods on sport}

Empirical sport science can tell us a lot about different measurable facts from physics, chemistry, biology, sociology etc. With its focus on empirical and mathematical method of research it can offer a lot of facts about physically measurable variables like time of activity, distance covered, angle of joints, speed of locomotion, muscular power and endurance, type of muscle fibre, concentration of oxygen in blood and its consumption in muscles, as well as the percentage of ball possession in a game, the number of shoots to the goal, the number of assistances, goals and faults etc. The object of empirical natural science can be anything that can be measured and then presented in numbers (statistics). This can be called naturalistic (Malik, 2006, p. 176) viewpoint on sport. Similar in empirical social sciences we can measure the facts like the percentage of sport active population, the average frequency of sport activities and their duration, statistically important differences between countries, the opinions of people about the value of sport etc. To be scientific we try to "think ourselves away" (Bell, 2009, p. 28) and keep objective viewpoint on sport. Therefore social science often reduces the knowledge of sport to its facts and analysis of its function in society, about how sport "works" for a particular group or individual. Functionalistic view on sport tries to explain sport in the frame of maintaining individual and social balance, the opportunity for individual self-expression, or for national or cultural preferences (Bell, 2009, p. 30). This approach includes in some degree the understanding of concepts used, however it is instrumental and often reduce sport to mere function or try to understand sport as function in cause-effect relations. While the questions about the nature and essence of sport, about its purpose and moral value stay untouched.

Phenomenon of sport is given to us in different possible experiences: objective empirical quantitative one and qualitative one. Both are important: Social and natural sciences try to understand how different things work from outside: how sport functions in human society, how body functions under unusual or extreme conditions in sport activities or competitions. Their approach is mainly quantitative and descriptive. They try to be objective, therefore also the objects of their research are reduced to measurable objects. On the other hand human sciences try to understand what is sport, what is (morally) good sport, what is its meaning and why. It acknowledges that it is not possible to separate sport from human being, so also the method of research must take into account the reality of a living person. It tries to explain sport from inside. Its approach is more qualitative and can be normative.

Is any of the scientific methods mentioned more appropriate for the research of sport? Some would say that only empirical, so called "hard sciences" are. But the others would oppose because they are reductionistic in their nature. Beside that it is not possible to prove with empirical method that empirical method is correct. If any method tries to prove that it is correct using its own method, it falls into the error of circular argumentation, because it lies on the presumption that it is right, but this must be first proved. A fundamental belief in any method is needed for the initial acceptance of any method. And wider knowledge about different methods can help in reasonable valuation of them. 


\section{Limitations of sport science as a natural and social science}

The type of method determines the results. If we use physical methods in the sphere of life, morality, art or culture in general, then also the results will be physical facts. But the life, morality, art or culture are much more then only physical objects. If we use only empirical methods in the sphere of sport, then also the results will be only empirical facts. To show the limits of scientific method we can point out some fundamental concepts and ideas in sport science, which do not derive from empirical measurement but from humanistic reflection and debates, e.g. concepts of training, competition, fitness, health, play, fair play etc. But, what are training, competition, fitness, health, play and fair play? If we want to make a correct interpretation of measured data, we must first analyse, understand and agree about the meaning of these concepts (McNamee, 2005, p. 1). None of these concepts can be defined empirically by measurement; they are all the result of discussions and agreements between rational subjects, persons. Only after we give meaning to them, the usage of these concepts has any sense in sport science. Therefore also sport training, usually conceived as a part of "hard" sport science, a kind of natural science dealing with body in extreme conditions, exceeds its borders. Behind sport training there is usually a kind of "philosophy" which operates with special concepts and ideas that have to be understood. A "philosophical" or theoretical (hypothetical) models of sport training are often supported partly with scientific discoveries, and partly with personal feelings of coaches, therefore sport training is often conceived as half science and half art. When natural or social scientist (e.g. coach) starts to interpret empirical results of researches (e.g. training activities), he leaves empirical methodology and arrives on the field of non-empirical methodology, which is more common for human sciences. Wittgenstein once clearly warned about using experimental method while neglecting understanding of used concepts. He said:

"For in psychology there are experimental methods and conceptual confusion. /.../ The existence of the experimental method makes us think that we have the means of solving the problems which trouble us; though problem and method pass one another by" (Wittgenstein, 1986, p. 232).

Let's take the concept of play. What is play and what makes some activity playful is out of reach of quantitative empirical sciences. It is only possible to measure the part of visible outcome of it, usually presented in the statistics at the end of game. But statistics cannot fulfil the expectations of actors or spectators: they want to feel and see real play/game with all its uncertainty; they want to know the reasons, the process which brings to the statistical facts. Problem that occurs in objective empirical and mathematical sciences is the objectification and determination of results, persons and life as such. But human being experiences self as a free being. Also sport without freedom will not be sport any more. It is widely accepted that one of the intrinsic value of sport is its freedom (Reid, 2012, p. 192). On the other hand it is widely accepted enlightenment tenet that use of narrow reason of "hard" sciences will liberate us. Not the truth, but the science itself will make as free. But it seems that narrow reason cannot acknowledge what can be experienced in sport. Contrary, narrow reason based on empirical and mathematical methodology always leads us to fixed results: $2+3$ equals 5 ; the athlete is $1,8 \mathrm{~m}$ high and his jump was $8,02 \mathrm{~m}$ long. There is no place to argue about this. Scientific reason literally coerces us to accept its conclusions. However, in sport activity we experience freedom. Spectators enthusiastically watch sport competitions because they believe that the athletes are free and the result is not fixed in advance. If that will not be the case, if empirical science could explain everything, sport would become absolutely uninteresting. The results of competition would be predicted in advance: you would just need to know the results of preliminary tests of physical fitness of athletes or teams. However, the experience shows us that in sport competitions the winner is not always the same person as the one who have the best results on different physical tests: the athlete with the best level of physical fitness is not automatically the winner in competition. Empirical facts about the abilities of athletes are important but not sufficient for the prediction of competition results.

Focusing on empirical methodological approach toward sport can lead to either extremely dualistic or materialistic conception of reality, athletes and sport as such. There is no place for human being as person, for his innate dignity. Just measurement of physical dimensions of body remains. Furthermore, purely 
mechanistic doctrine with its view of the "body-machine" working under the strict dictates of mechanical laws, leads to competitive concept of sport: because two bodies cannot occupy the same space at the same time this inevitable result in competition among them. Empirical measurement of physical quantities like time, distance or weight just add to the competitive nature and understanding of sport inside methodological approach of natural sciences.

With natural sciences technology in connected. Technology is usually understood as applied science (Kroes, 1998). With technology human beings try to transform and surpass nature. In sport technology is an means to progress in sport achievements. However many problems arise at this point: First, it is the question what is progress? Is progress just to go higher, faster and stronger? The meaning of progress is in great detail dependent on the chosen methodological approach toward sport. It can be progress in empirical quantitative facts about human body and its achievements, or can be progress in more qualitative dimensions of human existence as a whole. The other is moral problem: liberating human being and sport from nature as a model (when mentality of technology as transformation of nature is accepted) the nature as a normative concept in moral sense is lost. Consequences of this shift unavoidable lead to some kind of moral relativism because it seems that human thoughts alone cannot assure universal moral laws. Talking about fair play and other moral concepts in sport would not have sense any more. Moreover, empirical methodological approach has leading to reduced moral responsibility of agents. Human beings are reduced to mere workers who perform their duties without regard to the consequences for morality. In sport this can be seen in the problem of doping, which is from the technological point of view just a problem how to reach higher, run faster and perform stronger, without regard to the moral value of acts.

Absolutization of quantitative empirical scientific method in the sphere of sport science can result in destruction of sport itself. For example: if we want to measure dance, what we get out is anything but not dance. The paradox is when we want to research sport, at the same moment we destroy it. Like the perfect photography of a movement is not movement any more. It is like frozen. It necessarily misses the essence of movement! Parry shows this on the example of football:

"If you wish, you could measure the positions of all players on a football pitch when a certain variety of 'good pass' is made, the speed and direction of movement of players and ball, and so on. Let us say that this will be a scientific description of defence-splitting passes. Now, much may be learned from a scientific account, but one thing for certain will not be learned: and that is why it is a good pass. We already have to identify the good passes in order to know which ones to measure, so measurements of them do not constitute our knowledge of them but rather presuppose it. We know what counts as a good defence-splitting pass because we understand what football is and what reasons are operable within it. He who knows nothing of science may know more about football” (Parry, 2005, p. 29).

Natural and social sciences derive from empirical facts. But facts alone are without meaning. They get meaning when we interpret them. Therefore naturalism with its empirical and mathematical method excluding other methods is insufficient in the research of concepts, human persons or sport. When particular sciences exceed boundaries limited with their method, they become ideologies. This opens also the problem of dialog among different sciences. As realized Kosiewicz the:

"Representatives of natural, and especially biological, sport science cannot understand the sense of the existence of the humanities - including those dealing with sport (or physical culture) - which do not use empirical methodology based on experiment and observation" (Kosiewicz, 2010, p. 77).

Therefore it is necessary to know own and others limitations and potentials. The need for human science in sport is based on essential characteristics of sport itself - sport itself is nothing physical: it is an idea about a special kind of human movement. Although someday the empirical sciences will manage to explain every details in human bodily movement, the questions "what is sport?"; "why sport?"; "what is its 
meaning?" and "what is good sport?" will remain inaccessible for just empirical and mathematical approaches toward sport.

\section{The need for human sciences}

The method of research in great extend determines the way and contents of our cognitions. A biomechanics will in specific sport bodily movement see primarily speed, forces, power, angels, accelerations and resistance. But for ordinary people the same human bodily movement will be, for example, first of all a successful and beautiful ski jump. In this sense the approach obviously determines the content of our cognitions and understandings. Therefore it is clear that the question about the truth cannot be in exclusion of one or another approach (objective empirical and mathematical or humanistic reflection). It is true that in the case of human bodily movement there are such variables as speed, forces, power, angels, accelerations and resistance, however it is also true that the meaning of all this empirical facts can be only recognized if we understand this movement as a ski jump. The meaning of this movement - that it is a ski jump - is inaccessible to mere empirical-mathematical scientific method. We must understand the concept of ski jump. Therefore many very important areas of human life (the meaning, ethics, freedom, dignity, art, culture, religion or spirituality etc.) remain inaccessible to natural or social scientific method. It is truth that science can get to know truth, but not all the truth. The fundamental question in the search for truth concerns the consideration about the approach which is suitable for a chosen phenomenon. It must as much as possible keep the phenomenon to be faithfully what it is on its own. Scientific quantitative researching is not false, but it may be unsuitable for the chosen phenomenon (Klun, 2012, p. 74). For example, the phenomenon of death is much wider than the scientific concept of death (the cessation of all biological functions that sustain a particular living organism). Instead of allowing phenomenon to be what it is, we often reduce it to some partial viewpoint, as in the case when we reduce ski jump to the facts about speed, force, power etc. In searching for truth in sport it is necessary to try to allow sport to be what it is when it is practiced, when it is "alive".

Because of tight connection with human life sport also develops some values, but their goodness or badness is affair of discussion among rational beings and cannot be empirically measured or calculated. In ethics and fair play we speak about "ought" sentences. Rather than just describing how people treat each other, ethics should be about setting out appropriate behaviours or norms about how we ought to behave. But oughts could not be recognized as empirical facts: they are not the results of measuring of "public opinion" about sport. They can be contrary to what majority of people think about sport. However they are usually the result of discussion between rational subjects, human moral sense, conscience and tradition.

Sport is a phenomenon with great emotional influence on people. Therefore reduction to empirical and mathematical approach would miss its wholeness. Because people (as athletes or spectators) are inside of sport phenomenon, it is not possible to adequately research sport as an object out of us or in front of us. We are inside the flow of sport, just like we are inside the flow of our life. And it is not possible to step out of life, to stop it and objectively research it and understand it. Sport is always inside of life, sport is "happening" in the acts of person, therefore holistic approach to sport must take this into considerations.

The self being substance of sport - the human being as a person - is neither reachable to the empirical and mathematical method of modern science. What is left for empirical sport science is physical body: it can be measured scientifically, while the other part (mind, human being as a person) cannot. Therefore limitation of sport science to empirically measurable dimensions limit sport science to just one part of whole reality of sport. If we limit research to the sphere of empirical sciences, the human being is the one who is deprived. All questions about the essence, meaning and aim of sport, about ethics, aesthetic and fair play must be out of scientific reason and pushed into subjectivity and relativity. 


\section{Limitations of human sciences in sport}

Human sciences can go beyond natural and social sciences and can answer questions that cannot be reached by mathematical or empirical methods themselves, however they have some hidden problems which must be considered when applied to sport. First of them are fallacies in reasoning. Prerequisite for any reasoning is to understand and comprehend concepts and ideas correctly. The error can appear in any stage of three acts of the mind: understanding, judging and reasoning (Kreeft, 2008, p. 33). Among other important problems in human sciences is one known as "appeal to authority". It is in human character to conform itself (its ideas, thinking and findings) to dominant theories or ideologies. It can be recognized that researchers their findings often interpret in a way to confirm prevailing theories, which can be two edge sword: on one hand it can assure that findings will be widely accepted in scientific community, published in important journals and therefore even strengthening dominant theory, but on the other hand this can cover the real problems and hinder long-term scientific progress. This is even more important because of the fact that for verifying humanistic ideas and concepts, theories or ideologies is usually needed much more time (even years or centuries) than for verifying results of empirical experiments. The consequences of false theories or ideologies can therefore stay hidden in social community for ages, and can be even more dangerous than mere technological inventions. In last centuries much more people died because of social experiments i.e. application of different ideologies into society, than because of technological experiments. Therefore it is wise to ask which are prevailing ideas and concepts of sport in modern societies and how they will influence our future lives.

\section{Conclusion}

Sport science is fast growing sphere of research. As in any search for truth, also in sport science it is important to use appropriate methods regarding the object of research, because the methods in advance determine the results. In paper we have shown how different sciences (natural, social, human) with their methodological approaches toward sport have deep and powerful consequences not just for understanding of sport in academic community but also for the constitution of sport as is presented in everyday public life.

\section{REFERENCES}

Bell, B. (2009). Sport Studies. Exeter: Learning Matters Ltd.

Hosta, M. (2005). Poskus konceptualizacije športa /Conceptualization of sport/. Anthropos (1-4), 183-190.

Hoyrup, J. (2000). Human Sciences: Reappraising the Humanities Through History and Philosophy. New York: State University of New York Press.

Klun, B. (2012). Fenomenologija duhovnega življenja /Phenomenology of spiritual life/. In Repar, P., Repar, S., Božič, A. (Eds.). Udejanjanje duhovnosti v sodobnem svetu /Spirituality in modern world/ (pp. 69-82). Ljubljana: Kud Apokalipsa.

Kosiewicz, J. (2010). Sport and Philosophy - from Methodology to Ethics. Warsaw: BK Wydawnictwo.

Kreeft, P. (2008). Socratic logic. Indiana: St. Augustine's Press.

Kroes, P. (1998). Philosophy of Technology. In: Routledge Encyclopedia of Philosophy, Version 1.0, London: Routledge.

Malik, K. (2006). What science can and cannot tell us about human nature. In Wells, R.H., McFadden, J. (Eds.), Human nature: Fact and Fiction (pp.164-183). London: Continuum.

McFee, G. (2004). Sport, Rules and Values. London: Routledge.

McNamee, M. (2005). Positivism, Popper and Paradigms: an introductory essay in the philosophy of science. In McNamee, M. (Ed.), Philosophy and the Sciences of Exercise, Health and Sport (pp. 1-19). London: Routledge.

Parry, J. (2005). Must scientists think philosophically about science. In McNamee, M. (Ed.), Philosophy and the Sciences of Exercise, Health and Sport (pp. 20-31). London: Routledge. 
Ratzinger, J. (2006). Faith, Reason and the University: Memories and Reflections. Retrieved July 26, 2013, from http://www.vatican.va/holy_father/benedict_xvi/speeches/2006/september/documents/hf_ben-

Xvi_spe_20060912_university-regensburg_en.html

Reid, L.H. (2012). Introduction to the philosophy of sport. New York: Rowman \& Littlefield Publishers.

Ryal, E. (2011). The notion of a science of sport: some conceptual considerations. In Schulz, H., Wright, P.R., Hauser, T. (Eds.), Exercise, sports and health (pp. 171-176). Chemnitz: University of technology.

Wittgenstein, L. (1986). Philosophical investigations. Oxford: Basil Blackwell.

\section{AUTHOR'S ADDRESS: Jernej Pisk}

St. Stanislav's Institution, Ljubljana

Srednja vas 21, 4223 Poljane, Slovenia

Email: jernej.pisk@gmail.com 